\title{
Charge transport and microstructure in PFO:MEH-PPV polymer blend thin films
}

\author{
Manisha Bajpai ${ }^{\text {a,b}}$, Ritu Srivastava ${ }^{\text {a,*, }}$, M.N. Kamalasanan ${ }^{\text {a }}$, R.S. Tiwari ${ }^{\text {b }}$, Suresh Chand ${ }^{\mathrm{a}}$ \\ ${ }^{a}$ Centre for Organic Electronics, National Physical Laboratory (Council of Scientific and Industrial Research), Dr. K. S. Krishnan Road, New Delhi 110012, India \\ ${ }^{\mathrm{b}}$ Department of Physics, Banaras Hindu University, Varanasi 221005, India
}

\begin{abstract}
A B S T R A C T
Current density-voltage characteristics of poly(9,9'-dihexyl fluorenyl-2,7-diyl) (PFO) thin films $(\sim 120 \mathrm{~nm})$ have been studied in hole only device configuration at different temperatures $(100-290 \mathrm{~K})$ in pure form and with blending $(\sim 0.25-50 \mathrm{wt} \%)$ of poly(2-methoxy-5-(2-ethylhexyloxy)-1, 4phenylenevinylene) (MEH-PPV). It has been found that in the case of pure PFO the charge transport at low fields show an ohmic region which is followed by space charge limited conduction region. Blending of PFO with MEH-PPV at low ratio (up to $2 \mathrm{wt} \%$ ) increases the current density. As the ratio is increased further, the current density decreases. Analysis show that there is a change in conduction mechanism up to $6 \mathrm{~V}$ from SCLC to thermally activated ohmic conduction upon blending indicating the creation of new energy level near the transport states of PFO. Morphology of the polymer blended thin films was obtained by atomic force microscopy (AFM) technique. It has been found that the surface roughness of the investigated films is significantly increased upon blending indicating aggregation as well as phase separation at high blending ratios. The decrease in conductivity at high blending ratio can be related to the change in morphology of the films.
\end{abstract}

\section{Introduction}

Conjugated polymers are the promising candidates for light emitting applications in the context of their technological advantages such as flexibility, good solubility, ease of fabrication and processability. Polyfluorenes (PFs) and their derivatives are the most favoured materials for the fabrication of polymer light emitting diodes (PLEDs) and polymer photovoltaic devices due to their high lumineous efficiency and high carrier mobility [1-3]. The polyfluorenes also have an additional advantage of its emission in the bluish green region. Presently, poly (9,9'-dihexyl fluorenyl2,7-diyl) (PFO) is the most intensively investigated polymeric material for the single-layer organic electronic device applications [4-6]. Recent trend [7-9] to achieve improved efficiency is the use of single emissive layer made from the blend of polymers. However, performance of these devices depends primarily on the balanced transport of charge carriers. Therefore, to improve further the performance of LEDs based on PFO, there is a need to find innovative ways, either intrinsic or extrinsic, to tailor hole mobility in them, i.e., reduce it to balance with the low electron mobilities in these materials and thus achieve centralised and efficient radiative recombination for light emission. In single-layer devices better carrier balance has been achieved by using a blocking contact for hole injection and/or by mixing the polymer with one of the few available electron-transporting polymers $[10,11]$. Another solution is to reduce the hole mobility by doping the polymer with a hole-trapping polymer or small molecule such as, poly (2-methoxy-5-(2-ethylhexyloxy)-1, 4-phenylenevinylene) (MEH-PPV) and tetraphenylporphyrin (TPP). However space charge limited conduction (SCLC) in PFO and MEH-PPV has been well studied $[12,13]$ but the blend system of PFO:MEH-PPV is not yet sufficiently studied for the improvement of their electronic properties such as hole mobility and trap density. Recently it has been reported that blending small quantity of MEH-PPV in PFO shows large enhancement in EL efficiencies which has been attributed to efficient Förster type energy transfer [14]. In this work, we have tried to investigate the electrical properties of the blend system to correlate the enhancement of optical and electrical properties. For this purpose we have studied the $J-V$ characteristics of thin films of PFO blended with different ratios of MEH-PPV (0-50 wt\%) at temperatures ranging from $100 \mathrm{~K}$ to $290 \mathrm{~K}$. The studies were conducted in hole only configuration viz. ITO/PEDOT:PSS/PFO:MEH-PPV/Au respectively to eliminate the effect of double injection. Our results shows that the blending of MEH-PPV in PFO at low blending ratio (up to $2 \mathrm{wt} \%$ ) increases the current density and as the concentration is increased further, the current density decreases this has been explained on the basis of reduction in the hole mobility by generation of new trap sites and opens the way for balanced transport 
and radiative recombination of the charge carriers to improve the performance of PLEDs. Interesting and important results obtained in these investigations are reported in this short communication.

\section{Experimental details}

The hole only devices were prepared under identical conditions for both pure PFO and blended PFO:MEH-PPV in hole only device configurations i.e., ITO $(\Phi \sim 4.8 \mathrm{eV}) /$ PEDOT:PSS $(85 \mathrm{~nm}) /$ PFO $(\sim 120 \mathrm{~nm}) / \mathrm{Au} \quad(\Phi \sim 5.2 \mathrm{eV})$ and ITO $(\Phi \sim 4.8 \mathrm{eV}) /$ PEDOT:PSS $(85 \mathrm{~nm}) / \mathrm{PFO}: \mathrm{MEH}-\mathrm{PPV}(\sim 120 \mathrm{~nm})(x) / \mathrm{Au}(\Phi \sim 5.2 \mathrm{eV})$ ( $x$ shows the blending ratio i.e., $0.25 \mathrm{wt} \%$ to $50 \mathrm{wt} \%$ ), as shown in Fig. 1 and were denoted as device A, device B, device C, device D, device E, device $F$ and device $G$ respectively. Devices were grown on glass slides precoated with transparent ITO with sheet resistance of $18 \Omega / \square$. Substrates were ultrasonically cleaned in detergent solution for about $10 \mathrm{~min}$, followed by rinsing it in deionised water. These were then boiled in acetone, trichloroethylene and propanol and dried in each step at $90^{\circ} \mathrm{C}$ for $10 \mathrm{~min}$. Thereafter a thin film (thickness $\sim 85 \mathrm{~nm}$ ) of PEDOT:PSS (Aldrich, USA) was spin-coated onto the pre-cleaned and plasma treated ITO coated glass substrates at $1500 \mathrm{rpm}$ and cured at $120^{\circ} \mathrm{C}$ for $30 \mathrm{~min}$ in vacuum. Subsequently, two homogeneous solutions were made i.e., by dissolving PFO in chlorobenzene with material content of $5 \mathrm{mg} / \mathrm{ml}$ and other by blending PFO and MEH-PPV (0.25-50 wt\%) in the same solvent. Thin films were prepared by spin coating a chlorobenzene based solution of PFO and blended solution of PFO:MEH-PPV on precured PEDOT:PSS coated glass substrates under nitrogen atmosphere in a glove box. These films were then annealed at $150^{\circ} \mathrm{C}$ in vacuum for $2 \mathrm{~h}$. and the film thicknesses were estimated by ellipsometry. On top of these films, Au electrodes $\sim 500 \mathrm{~nm}$ were deposited by vacuum thermal evaporation at vacuum of $5 \times 10^{-6}$ Torr. The devices so obtained were sealed using ultra-violet (UV) irradiated epoxy resin to inhibit atmospheric oxidation of electrodes. The $J-V$ characteristics of these samples in the temperature range of $100-290 \mathrm{~K}$ were measured using liquid nitrogen cooled cryostat coupled with a Keithley 2400 Source measure unit interfaced with a PC.

\section{Results and discussion}

Fig. 2 shows the $\log J-\log V$ characteristics of ITO/PEDOT:PSS/PFO/Au (Device A) has been studied at different temperatures in the range $100-290 \mathrm{~K}$. These curves can be divided into three distinct regions (I), (II) and (III). Region (I) is ohmic, where the current density is proportional to the voltage, region (II) is the SCLC limited by discrete traps where the current density is directly proportional to the square of the voltage and region (III) is the trap charge limited conduction where current increases exponentially and the onset of the trap-filled voltage

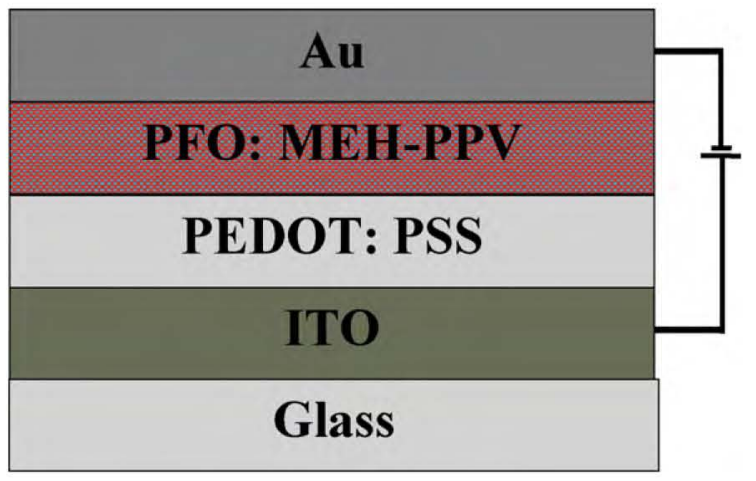

Fig. 1. Schematic diagram of the hole only device.

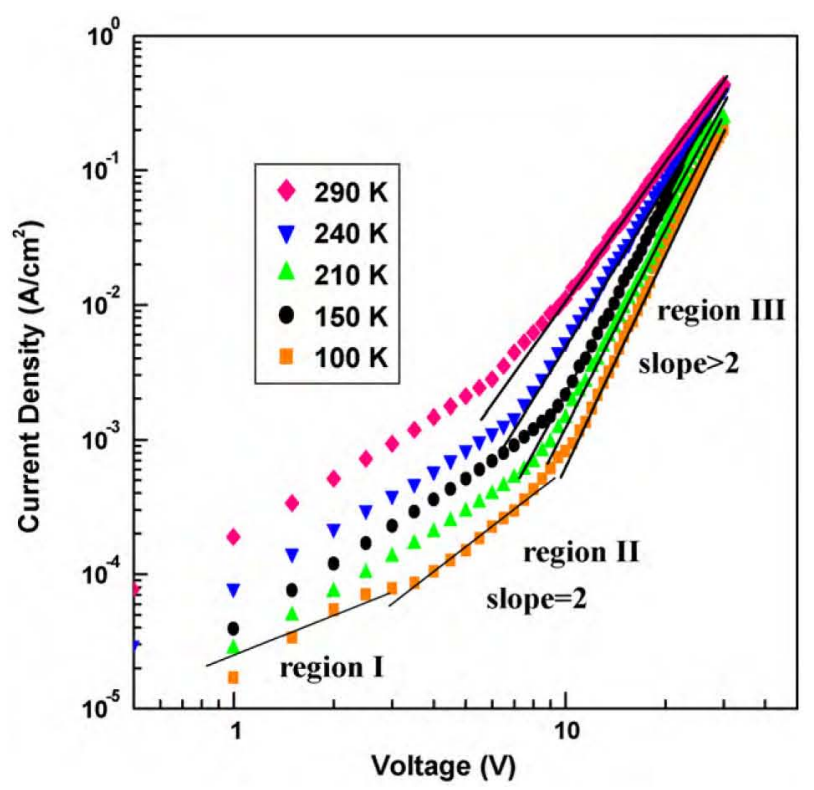

Fig. 2. $I-V$ characteristics of device $A$ at different temperatures in the range 290-100 K. Experimental data and theoretically generated curves are represented by symbols and solid lines using Eq. (5) respectively. The values of fitting parameters for both devices fitted with traps distributed SCLC models are tabulated in the text.

$V_{\text {TFL }}$ gives the density of traps Nt. Ideally, there must be an another region (IV) which is the trap-filled SCLC which is free from the influence of traps. But we could not observe this region due to the failure of device at higher voltages.

The low field ohmic region (region (I)) has been attributed due to the transport of intrinsic background carriers present inherently in the bulk of the polymer film and dominantly arising due to impurities, defects, etc. during the formation of the film. The transport of such carriers will dominate the $J-V$ behaviour of the polymer up to the values of the electric field where the injection effect does not come into play. However, as the injected hole density starts dominating the density of intrinsic background carriers the onset of nonlinearity occurs in the $J-V$ curves indicating thereby that the bulk non linear effects have started dominating the conduction. This is seen as an increase in the slope of the $J-V$ curves which become greater than 1 (region (II)). In the region (II), from $2 \mathrm{~V}$ to $7 \mathrm{~V}$, the current density shows a square law dependence on voltage indicating that the injection barrier is very small and the charge extraction rate is mainly limited by the bulk-transport characteristics rather than hole injection. The increase in current density with increase in temperature indicates the presence of discrete traps.

To understand the mechanism of region (III), the experimental data has been analyzed in terms of SCLC with exponential distribution of traps in energy space $[7,15,16]$. For this type of transport the $J-V$ characteristics can be described by trap charge limited model (TCLC) in region (III), in which, the distribution function for the trap density as a function of energy level $\mathrm{E}$ above the edge of HOMO and distance $x$ from the injecting contact for hole carriers can be described as follows:

$N(E)=N_{t}(E) S(x)$

where $N_{t}(E)$ and $S(x)$ represent the energy and spatial distribution function of traps and if the traps are distributed exponentially in the energy space within the forbidden gap, consequently the trap density is expected to be in the form of an exponential distribution at energies $E$ within the forbidden energy gap, such that

$N(E)=\left(\frac{H_{b}}{E_{t}}\right) \exp \left(\frac{E}{E_{t}}\right) S(x)$ 
where $H_{b}$ is the density of traps at the edge of valence band and $E_{t}$ is characteristic trap energy. $E_{t}$ is also often expressed in terms of the characteristic temperature of trap distribution $T_{c}$ For the case when the trap energy $E=E_{t}$, Eq. (2) becomes $N\left(E_{t}\right)=H_{b} / e S(x)$ which shows that $E_{t}$ characterizes the exponential trap distribution and defines the energy level where the trap density has been reduced by $1 / e$ of its value at the valence band edge. Hence the characteristic width of the exponential distribution is set by the value of $E_{t}$.

In case of such an exponential distribution of traps, assuming that the trapped hole carrier density $\left(p_{t}\right) \gg$ free hole carrier density $(p)$ and using continuity equation and boundary condition as follows:

$J=q \mu p(x) F(x)$

$V=\int_{0}^{d} F(x) d x$

the $J-V$ characteristic is governed by [17-19]:

$J=q^{1-l} \mu N_{v}\left(\frac{2 l+1}{l+1}\right)^{l+1}\left(\frac{l}{l+1} \frac{\varepsilon \varepsilon_{0}}{H_{b}}\right)^{l} \frac{V^{l+1}}{d^{2 l+1}}$

where $J$ is the current density, $V$ is the applied voltage, $q$ is the elementary charge, $d$ is the thickness of the material film, $\mu$ is the hole carrier mobility, and $F(x)$ is the electric field, $N_{v}$ is the effective density of states, $\varepsilon$ is the dielectric constant of material, $\varepsilon_{0}$ is permittivity of the free space. For a given thickness $J$ is proportional to $V^{l}$ where $l$ depends on device characteristics, indicating that the traps are distributed exponentially within the forbidden energy gap following Eq. (5). The parameter $l$ determines the distribution of traps in the band gap; $l=1$ corresponds to shallow trap behaviour with discrete energy. Values of $l$ greater than 2 signify the presence of exponential distribution of traps. The value of the characteristic temperature for trap distribution was obtained from the slope of the plot of 1 versus temperature.

The experimental data of device $A$ has been analyzed in terms of above equations from temperatures $100 \mathrm{~K}$ to $290 \mathrm{~K}$ as shown in Fig. 2. It is seen that the experimental data shows good agreement to the above mentioned trap distributed SCLC model. Here symbols represent the experimental data while solid lines correspond to the theoretically generated curves at corresponding temperatures using Eq. (5). As shown by solid lines in Fig. 2, the experimental data in temperature range from 100 to $290 \mathrm{~K}$ agrees well with the trap model with fitting parameters as $N_{v}=3.0 \times 10^{19} \mathrm{~cm}^{-3}, H_{b}=3.7 \times 10^{17} \mathrm{~cm}^{-3}$, $\mu=3.5 \times 10^{-5} \mathrm{~cm}^{2} \mathrm{~V}^{-1} \mathrm{~s}^{-1}, T_{C}=540 \mathrm{~K}$, and $E_{t}=46 \mathrm{meV}$.

To study the effect of blending of MEH-PPV on hole transport of PFO polymer we have fabricated the hole only device $B$, device $C$, device $D$, device $E$, device $F$ and device $G$ in configuration ITO/PEDOT:PSS/PFO:MEH-PPV $(x) / A u$ where $x$ varies from $x=0 \mathrm{wt} \%$, $0.25 \mathrm{wt} \%, 1 \mathrm{wt} \%, 2 \mathrm{wt} \%, 10 \mathrm{wt} \% 25 \mathrm{wt} \%$ and $50 \mathrm{wt} \%$. and studied the $J-V$ characteristics at room temperature as shown in Fig. 3. It has been observed that increasing the blending ratio of MEH-PPV from $0 \mathrm{wt} \%$ to $2 \mathrm{wt} \%$ enhances the current density gradually which is due to the decrease of the resistance and activation energy (shown in Fig. 4) of the PFO:MEH-PPV blend layer [20]. However, the current conduction is reduced slightly as the blending ratio further increases from $10 \mathrm{wt} \%$ up to $50 \mathrm{wt} \%$. This result may be attributed to the effect of carrier quench or increased disorder due to higher concentration of MEH-PPV (the current density versus wt\% concentration in the inset of Fig. 3 at voltage $1 \mathrm{~V}$ shows a quick increase in current density up to a $2 \mathrm{wt} \%$ and above $2 \mathrm{wt} \%$ the current density gradually decreases). The mechanism responsible for the variation of these charge transport parameters on different blending ratio can be understood (I) in terms their trapping behaviour analyzed from energy level diagram and (II) of their surface roughness by means of atomic force microscopy (AFM).

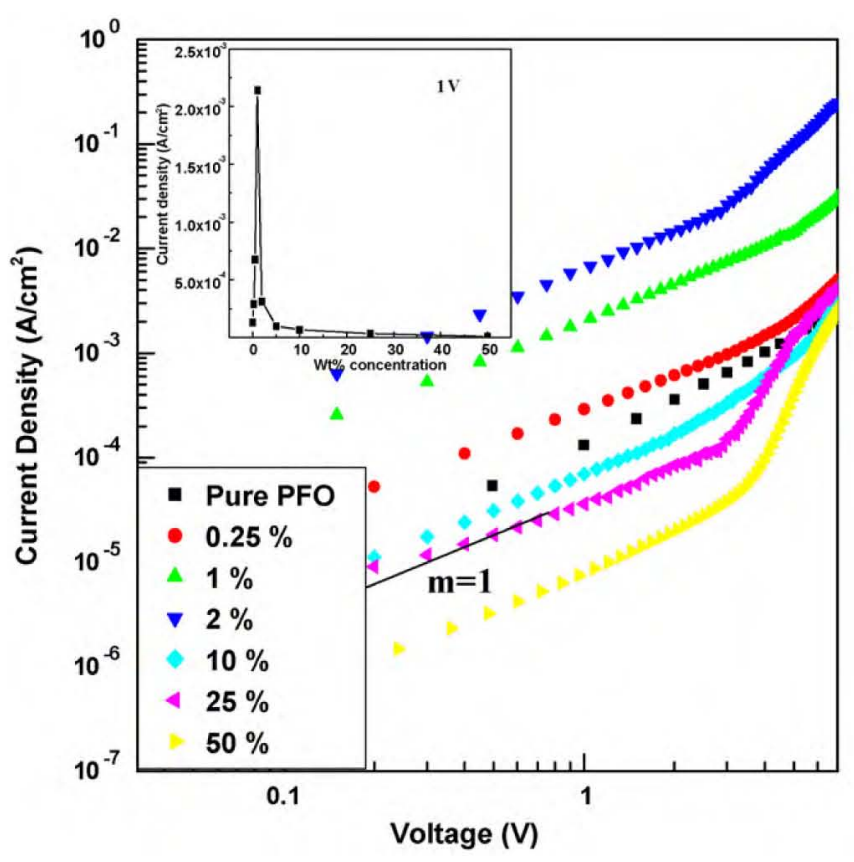

Fig. 3. Comparison of current density for device A, device B, device C, device D, device $\mathrm{E}$, device $\mathrm{F}$ and device $\mathrm{G}$ at a fixed temperature $\sim 290 \mathrm{~K}$ (inset shows current density versus blending concentration curve for all devices at a fixed voltage $\sim 1 \mathrm{~V}$ ).

It has been observed that the current density in the blend samples varies directly proportional to the voltage (ohmic conduction) up to $5 \mathrm{~V}$ (Fig. 3 ) in contrast to the behaviour of pure PFO film (device $\mathrm{A}$ ) in which the current was varying with square of applied voltage in this region. To understand this phenomenon we have studied the $J-V$ characteristics of the film blended with $2 \mathrm{wt} \% \mathrm{MEH}-$ PPV (device D) at different temperatures (100-290 K), shown in Fig. 5. It has been observed that (Fig. 5) up till $5 \mathrm{~V}$ the current varies linearly with applied voltage and decreases with decrease in temperature. The plot of current density against $1000 / \mathrm{T}$ is shown in the inset of Fig. 5, which was found to be straight line indicating thermally activated generation of free charge carriers. At

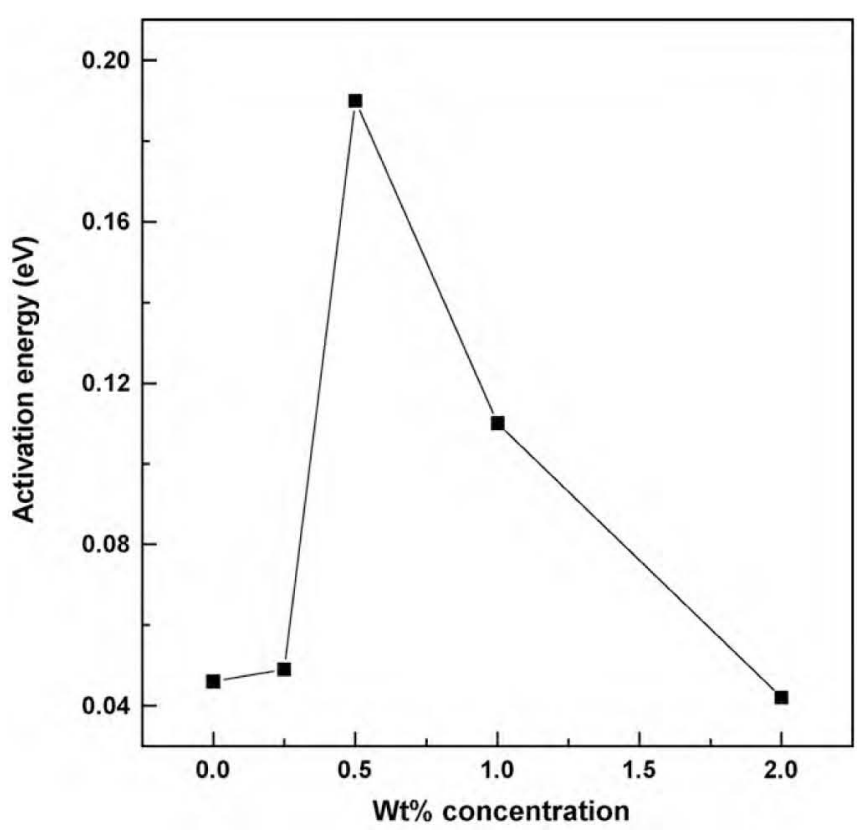

Fig. 4. Activation energy versus wt\% concentration plot (activation energy is calculated from the slop of the current density $(\ln J)$ versus temperature plot (figure not shown)). 
Values of transport parameters for the devices under investigation.

\begin{tabular}{|c|c|c|c|c|c|}
\hline $\begin{array}{l}\text { Transport } \\
\text { parameters }\end{array}$ & $\begin{array}{l}\text { Density of states } \\
\left(\mathrm{cm}^{-3}\right)\end{array}$ & $\begin{array}{l}\text { Density of traps } \\
\left(\mathrm{cm}^{-3}\right)\end{array}$ & $\begin{array}{l}\text { Characteristic } \\
\text { temperature (K) }\end{array}$ & $\begin{array}{l}\text { Characteristic trap } \\
\text { energy (meV) }\end{array}$ & $\begin{array}{l}\text { Hole mobility } \\
\left(\mathrm{cm}^{2} \mathrm{~V}^{-1} \mathrm{~s}^{-1}\right)\end{array}$ \\
\hline Device A & $3 \times 10^{19}$ & $3.7 \times 10^{17}$ & 540 & 46 & $3.5 \times 10^{-5}$ \\
\hline Device D & $3 \times 10^{19}$ & $2.8 \times 10^{17}$ & 690 & 33 & $1.9 \times 10^{-4}$ \\
\hline
\end{tabular}

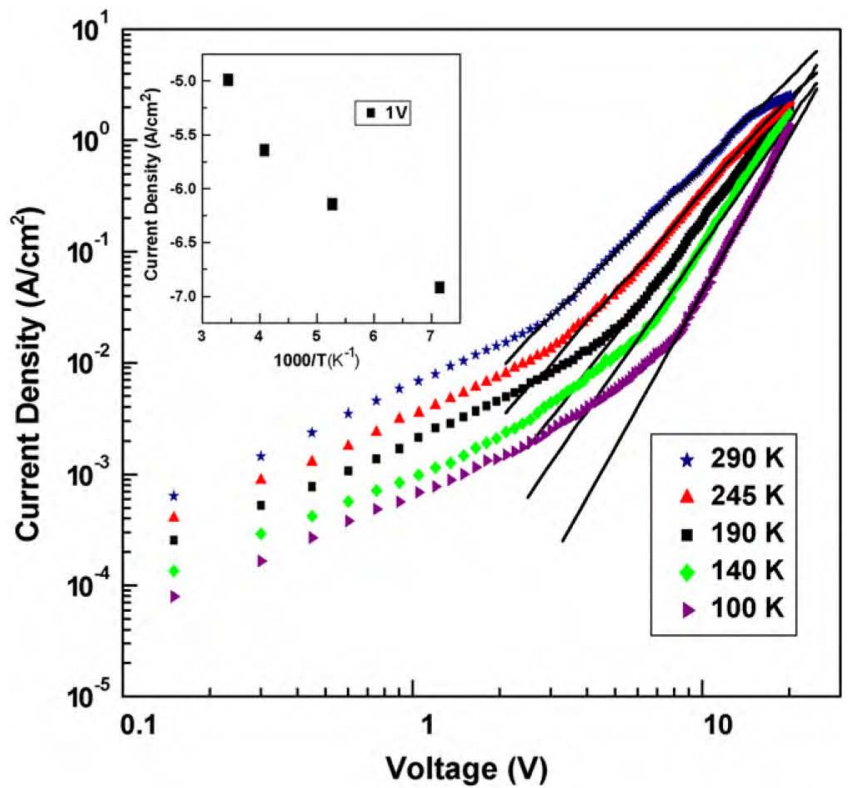

Fig. 5. $J-V$ characteristics of device $\mathrm{D}$ at different temperatures in the range 290-100 K. Experimental data and theoretically generated curves are represented by symbols and solid using Eq. (5) respectively. The values of fitting parameters for both devices fitted with traps distributed SCLC models are tabulated in the text. Inset shows the current density $(\ln J)$ versus temperature plot.

higher voltages the slope of $J-V$ characteristics show higher slope. This has been analyzed using trap model as mentioned above. Here it has been also found that the data fits very well with the trap model for all analyzed temperatures. The various transport parameters for device D, being $N_{v}=3.0 \times 10^{19}, H_{b}=2.8 \times 10^{17} \mathrm{~cm}^{-3}$, $\mu=1.9 \times 10^{-4} \mathrm{~cm}^{2} \mathrm{~V}^{-1} \mathrm{~s}^{-1}, T_{C}=690 \mathrm{~K}$, and $E_{t}=33 \mathrm{meV}$. The overall performances of all the devices are summarized in Table 1.

The ohmic region below $5 \mathrm{~V}$ can be explained from the energy level diagram of PFO and MEH-PPV as shown in Fig. 6, which shows a type-I (straddling type) heterojunction and holes injected. If we consider the relative HOMO position of MEH-PPV and PFO, there is a band offset of $0.5 \mathrm{eV}$ but since we observe thermally generated holes upon blending it seems that shallow energy levels are created during the process. This can be explained in the following way: when we mix MEH-PPV and PFO having different Fermi

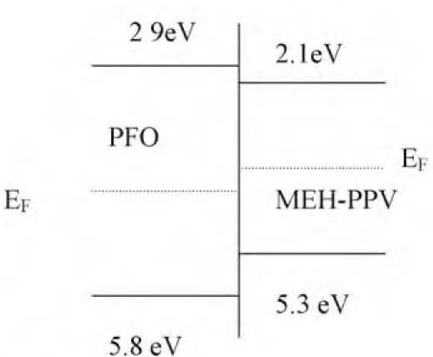

Before blending

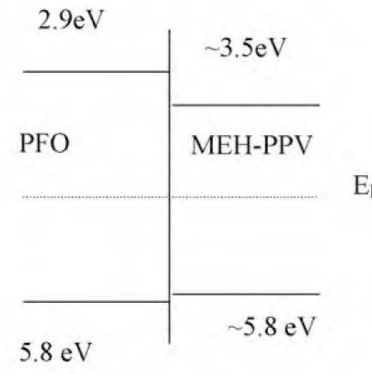

After blending
Fig. 6. Proposed energy level diagram of energy level alignment process when PFO and MEH-PPV form a heterojunction: (a) before blending and (b) after blending. levels, charge transfer between the two polymers will takes place to align the Fermi levels. This process may bring the HOMO of both MEH-PPV and PFO very close to each other (see Fig. 6). It is likely that in the low voltage region holes from the HOMO of the MEH-PPV gets thermalized and go to the transport states of the PFO thereby increasing the current. Since this process takes place without injection process we get ohmic conduction similar to impurity conduction in other semiconductors. As the blending ratio increases, the density of HOMO level also increases which explains the increase in current density with increasing blending ratio. At still higher concentration agglomerations/phase separation of the PFO and MEH-PPV takes place and since MEH-PPV regions have lower mobility the total current density decreases.

To further support these observations of the pure and blended film morphology were characterized by atomic force microscopy (AFM) in tapping mode. Fig. 7(a)-(c) shows AFM images for films
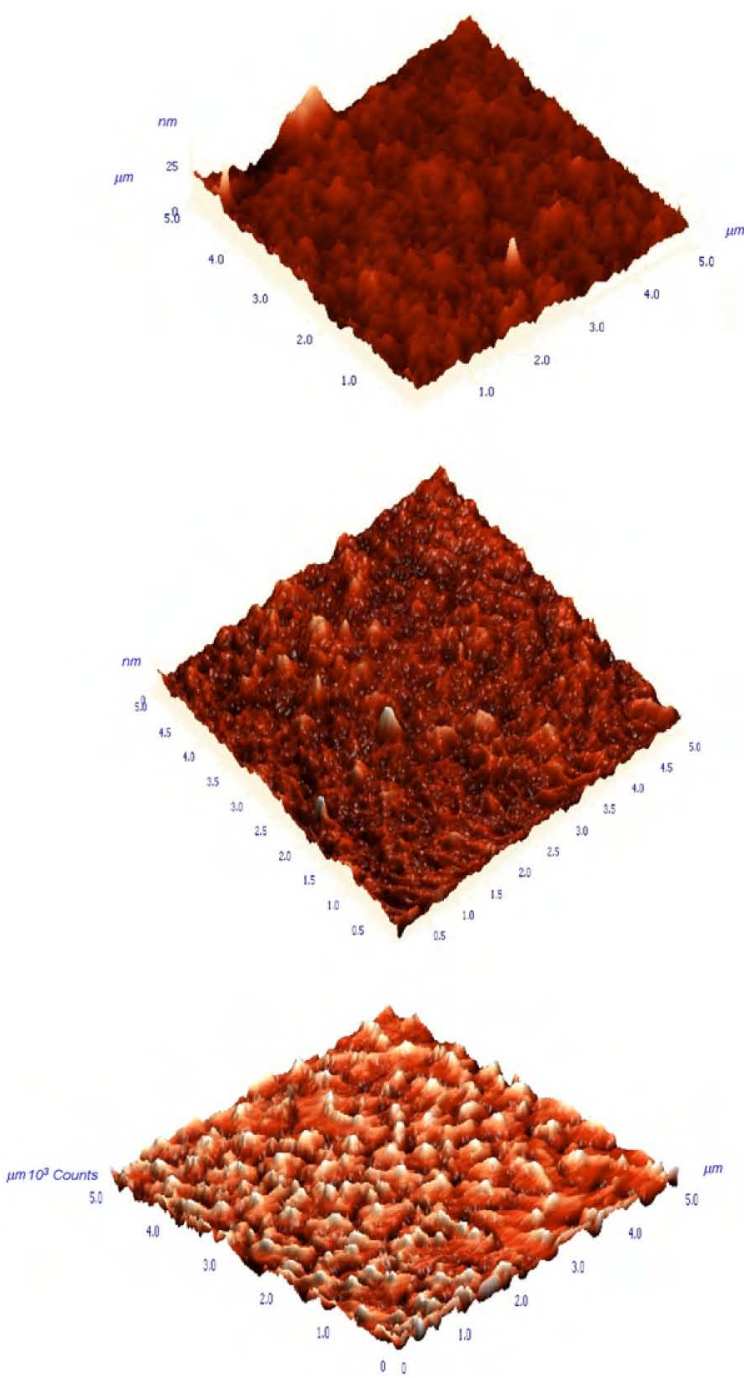

Fig. 7. AFM height images $(5 \mu \mathrm{m} \times 5 \mu \mathrm{m}$ size) of the surface of the active layer consisting of (a) as-prepared pure PFO, (b) $2 \mathrm{wt} \% \mathrm{MEH}-\mathrm{PPV}$ in PFO and (c) $25 \mathrm{wt} \%$ MEH-PPV in PFO. 
containing $0 \mathrm{wt} \%, 2 \mathrm{wt} \%$, and $25 \mathrm{wt} \%$ blend films. Both the RMS and Ra roughness increase when MEH-PPV is blended in PFO. The root mean square (RMS) of pristine PFO was found to be $2.31 \mathrm{~nm}$ whereas the blend film of $2 \mathrm{wt} \%$ of MEH-PPV shows the RMS of $1.34 \mathrm{~nm}$ and the RMS of the blend film of $25 \mathrm{wt} \%$ of MEH-PPV shows the RMS of $14.33 \mathrm{~nm}$. The average roughness (Ra) of pristine PFO was found to be $1.68 \mathrm{~nm}$ whereas the blend film of $2 \mathrm{wt} \%$ of MEHPPV shows the Ra of $1.11 \mathrm{~nm}$ and the Ra of the blend film of $25 \mathrm{wt} \%$ of MEH-PPV has reached up to $8.44 \mathrm{~nm}$.

Compared to pristine PFO, the blend films with $25 \mathrm{wt} \%$ blend film exhibit higher root mean square (RMS) roughness and average roughness $(\mathrm{Ra})$ values and associated with domains of different heights. This observation suggests that the relative roughness of the blend films at higher blending ratio can be attributed only to the influence of a strong MEH-PPV molecule aggregation, as more agglomeration would be expected for blends of higher MEH-PPV content which lead to decrease in further charge transport.

\section{Conclusions}

We have studied the effect of blending on the transport properties of PFO. It has been seen that the blending produces thermally generated carriers in the matrix. The current density increases up to $2 \mathrm{wt} \%$ and then strongly decreases. The decrease in the current density upon higher blending concentration has been attributed to disorder as well as agglomeration in the matrix which is supported by AFM studies.

\section{Acknowledgements}

The authors would like to thank and greatly acknowledge to Director, National Physical Laboratory India to encourage us for novel research and Council of Scientific and Industrial Research (CSIR), India for the financial support.

\section{References}

[1] Y.Y. Noh, D.Y. Kim, Y. Yoshida, K. Yase, B.J. Jung, E. Lim, H.K. Shim, R. Azumi, Appl. Phys. Lett. 85 (2004) 14-16.

[2] D. Neher, Macromol. Rapid Commun. 22 (2001) 1365.

[3] H.H. Fong, A. Papadimitratos, G.G. Malliaras, Appl. Phys. Lett. 89 (2006) 172116.

[4] G.K. Ho, H.F. Meng, S.C. Lin, S.F. Horng, C.S. Hsu, L.C. Chen, S.M. Chang, Appl. Phys. Lett. 85 (2004) 20.

[5] C.M. Yang, C.H. Wu, H.H. Liao, K.Y. Lai, H.P. Cheng, S.F. Horng, H.F. Meng, J.T. Shy, Appl. Phys. Lett. 90 (2007) 133509

[6] R.J.D. Vries, R. Coehoorn, S.L.M. van Mensfoort, V. Shabro, S.I.E. Vulto, R.A.J. Janssen, Appl. Phys. Lett. 94 (2009) 163307.

[7] M.M. Mandoc, F.B. Kooistra, J.C. Hummelen, B. de Boer, P.W.M. Blom, Appl. Phys. Lett. 91 (2007) 263505.

[8] A.J. Campbell, D.D.C. Bradley, H. Antoniadis, J. Appl. Phys. 89 (2001) 3343.

[9] A.J. Campbell, D.D.C. Bradley, H. Antoniadis, Appl. Phys. Lett. 79 (2001) 2133.

[10] A. Babel, S.A. Jenekhe, Macromolecules 36 (2003) 7759.

[11] D.F.O. Brien, C. Giebeler, R.B. Fletcher, A.J. Cadby, L.C. Palilis, D.G. Lidzey, P.A. Lane, D.D.C. Bradley, W. Blau, Synth. Met. 116 (2001) 379.

[12] M. Redecker, D.D.C. Bradley, M. Inbasekaran, E.P. Woo, Appl. phys. Lett. 73 (1998) 1565.

[13] A.J. Campbell, D.D.C. Bradley, D.G. Ledzey, J. Appl. Phys. 82 (1997) 6326.

[14] N. Ananthakrishnan, G. Padmanabham, S. Ramakrishnan, J.R. Reynolds, Macromolecules 38 (2005) 7660-7669.

[15] A.K. Kapoor, S.C. Jain, J. Pootmans, V. Kumar, R. Mertens, J. Appl. Phys. 92 (2002) 3835.

[16] N.F. Mott, R.W. Gurney, Electronic Processes in Ionic Crystals, Oxford, London, 1940.

[17] A.R. Brown, D.D.C. Bradley, J.H. Burroughes, R.H. Friend, N.C. Greenham, P.L. Burn, A.B. Holmes, A. Kraft, Appl. Phys. Lett. 61 (1992) 2793.

[18] V. Kumar, S.C. Jain, A.K. Kapoor, J. Pootmans, R. Mertens, J. Appl. Phys. 94 (2003) 1283.

[19] K.C. Kao, W. Hwang, Electrical Transport in Solids, Pergamon, Oxford, 1981, p. 160.

[20] M.T. Hsieh, C.C. Chang, J.F. Chen, C.H. Chen, Appl. Phys. Lett. 89 (2006) 103510. 